\title{
An Improved Algorithm Based on Wellner's Threshold Segmentation Method
}

\author{
Zhang Daode*, Ye Xuhui and Hu Xinyu
}

School of Mechanical Engineering, Hubei University of Technology, Wuhan 430068, China

\begin{abstract}
According to the Wellner algorithm whose speed is slow and the image threshold is not smooth for computing a large amount of defects, this paper presents two kinds of improved scheme, the first scheme is onedimensional smoothing algorithm (ODSA), the second is based on the first one and its name is integral image algorithm(IIA). The former one mainly considering the spatial relationship between pixels, to ensure the continuity of pixels after segmentation; the latter dynamically set local threshold according to different environmental, to avoid local all black or all white, to separated object from the background exactly. Through the contrast experiment, its results show that, Wellner algorithm is not ideal at the edge of image processing, and the time complexity is too high. The one-dimensional smoothing algorithm is clear and accurate when processing contour, but the time complexity is relatively large. When we use the integral image algorithm to processing image, the foreground and background segmentation is clear, and the error rate is very low, and the time complexity is minimum, and it has good ability to adapt to the scene, so the integral image algorithm is the best.
\end{abstract}

Keywords: Integral image, one-dimensional smoothing, segmentation, wellner algorithm.

\section{INTRODUCTION}

Image segmentation is not only a classical difficult problem in image processing, but also the basic technique in image processing and computer vision field. Its desire to separate objects from the background in the image, to extract the interesting target image from the background, to provide the basis for follow-up treatment [1]. The threshold segmentation method become the fundamental segmentation technology [2] and widely used because of its simple realization, small amount of calculation, stable performance. Threshold selection method can be divided into two categories, one is the global threshold method and the other is local threshold method. The global threshold method can handle the image well which the lighting conditions is uniform or the light does not change rapidly, so it perform poorly in opened office or industrial site. But the local threshold segmentation method, such as fuzzy threshold segmentation [3, 4], can make up for the defects, and have a stronger ability to adapt to the scene. This paper proposes an improved adaptive threshold segmentation algorithm, processing the image under the effect of light in the open environment and human interference. It dynamic set local threshold to obtain good segmentation results.

\section{ANALYSIS OF WELLNER ALGORITHM}

\subsection{Theory of Wellner Algorithm}

In 1979, R. J. Wall developed An algorithm to dynamically calculate the threshold [5] according to the background luminance. According to the principle of Wall algorithm, on account of an image which the size is $\mathrm{M}^{*} \mathrm{~N}$ and the gray level is L, the specific steps of the algorithm are as follows:

(1) Firstly, divided the image into smaller blocks, $\mathrm{X}_{i}, i \subset[0, n)$

(2) Calculate histogram of each block orderly, $\mathrm{H}_{i}, i \subset[0, n)$

(3) Calculate threshold of each block according to corresponding peak value of each histogram. The threshold is $\mathrm{u}_{i}, i \subset[0, n)$

(4) Each pixel threshold interpolation obtained according to adjacent block threshold

(5) Binaryzation according to the threshold value from step (4)

This step (3) is the key point, for each small block, the processing step are as follow:

Suppose that we get an image which original gray level is $\mathrm{M}$, so the range of gray level is $(0, \mathrm{M}]$. The account of gray level $\mathrm{i}$ is $\mathrm{n}_{i}$. Then normalize the gray value:

$\mathrm{P}_{i}=\frac{n_{i}}{M}$

Suppose that the segmentation threshold is $t$, it divided the gray image into two categories [6]: foreground and background. The ratio of foreground is $\mathrm{w}_{0}$, the average gray level is $\mathrm{u}_{0}$. The ratio of background is $\mathrm{w}_{1}$, the average gray level is $\mathrm{u}_{1}$, and the total average value is $\mathrm{u}_{T}$ 
The variance between classes is defined:

$\sigma^{2}=\mathrm{w}_{0} *\left(u_{0}-u_{T}\right) *\left(u_{0}-u_{T}\right)+w_{1} *\left(u_{1}-u_{T}\right) *\left(u_{1}-u_{T}\right)$

Simplify the above formula, then we get

$\sigma^{2}=\mathrm{w}_{0} * w_{1} *\left(u_{0}-u_{T}\right) *\left(u_{T}-u_{1}\right)$

$\mathrm{w}_{0}=\sum_{i=0}^{t} P_{i}$

$\mathrm{w}_{1}=\sum_{i=t+1}^{M-1} P_{i}$,

$\mu(\mathrm{t})=\sum_{i=0}^{t} P_{i} *_{i}$

$\mu_{\mathrm{T}}(t)=\sum_{i=t+1}^{M-1} P_{i} * i$

$\mathrm{u}_{0}=\frac{\mu(t)}{w_{0}}$,

$\mathrm{u}_{1}=\frac{\mu_{T}(t)}{w_{1}}$,

$\mathrm{u}_{T}=\mu(t)+\mu_{T}(t)$

When we traverse $\mathrm{T}$ in range $[1, \mathrm{M}]$, the one that prompt $\sigma^{2}$ biggest is the best segmentation value.

\subsection{Result Analysis of Wellner Algorithm}

Fig. (1). shows the text image under the light of office, it has the following characteristics: there are the image edge with a light source, a black text on a white background, a gray text on a white background, different shades and a very thin horizontal black line under the word "PaperWorks". In Fig. (2), according to the Wellner algorithm effect diagram we can see that: the left side is closest from the source, some letters of the edge is not clear; the black stripes above "PaperWorks" is obvious narrow, and the intermediate part is partially broken; the right black bar disappears completely, the reason is that the part of light is weakest, so it occur errors; the line under Paperworks is not obvious; the gray text appears some holes. Fig. (3). is a board, and the board image

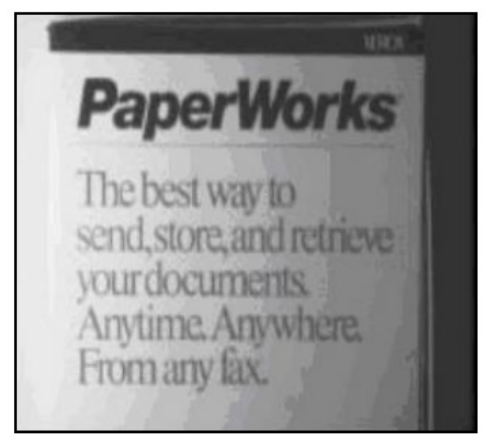

Fig. (1). Original text image.

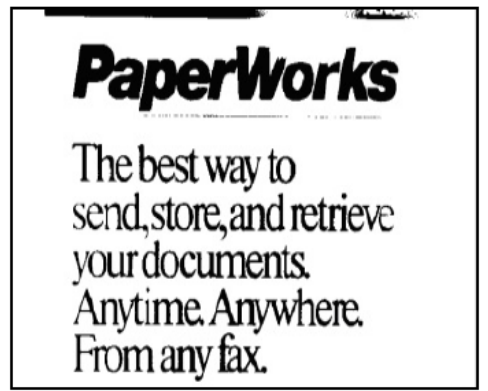

Fig. (2). Result of Wellner algorithm.

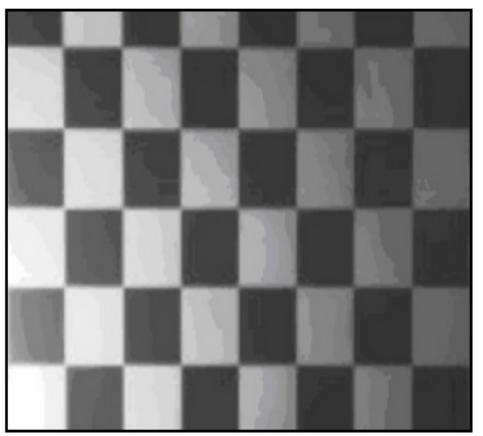

Fig. (3). The original board image.

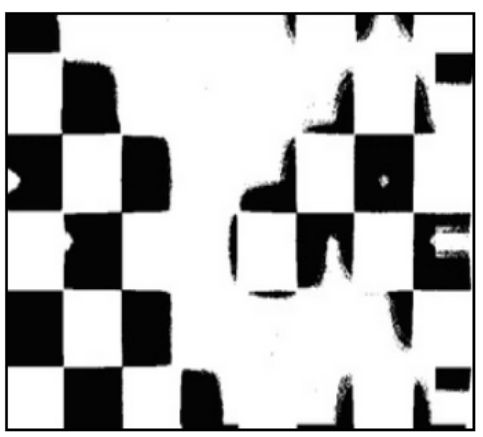

Fig. (4). Result of Wellner algorithm.

is divided into 9 blocks $(3 * 3)$. We select threshold which is $80 \%$ of the peak value. For the local histogram may be cheated by a lot of black or white point area, it will lead to the change of threshold is not smooth in the whole image, and the results may be very terrible, as we can see in Fig. (4).

To sum up: the error rate of Wellner algorithm is high, and the treatment is ineffective, so the algorithm needs to be further improved.

\section{IMPROVED ALGORITHM BASED ON WELLNER SEGMENTATION ALRORITHM}

\subsection{The Improved Algorithm Based on One-Dimensional} Smoothing Method

\subsubsection{The One-Dimensional Smoothing Algorithm Princi- ple and Implementation}

Most literature algorithms [7] are complex than Wellner algorithm, therefore they requires much more running time. Aiming at these problems, this paper presents a fast adaptive threshold algorithm based on Wellner algorithm. The basic idea of the algorithm is to traverse the image, calculate a 


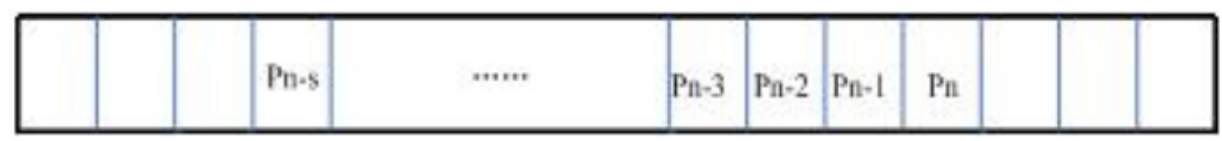

Fig. (5). s pixels after point $n$.

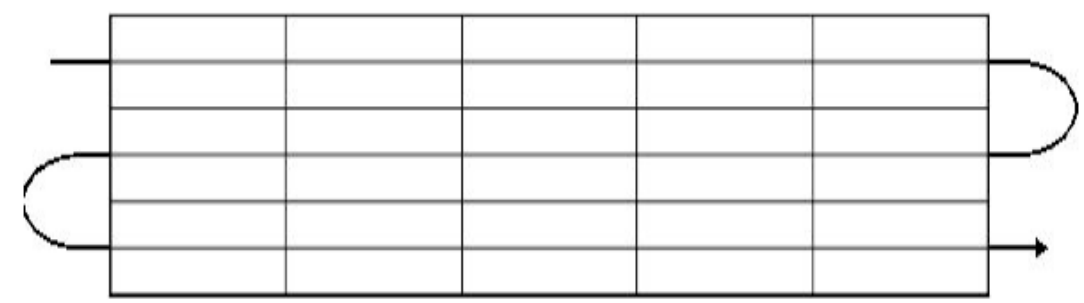

Fig. (6). Scanning mode of ODSA.

moving average, and if the pixel is obviously lower than the average value then set it to black, otherwise set it to white.

A image, the size is $\mathrm{M}^{*} \mathrm{~N}$, and the gray level is $\mathrm{L}$, set $\mathrm{P}_{n}$ the pixel gray value [8] at a point $n$. we assume that the image is a single by all rows sequentially connected, and set $\mathrm{f}_{s}(n)$ is the sum of $\mathrm{N}$ pixels at the last $\mathrm{s}$ point. Fig. shows the case that $\mathrm{s}$ pixels after point $\mathrm{n}$.

$\mathrm{f}_{s}(n)=\sum_{i=0}^{s-1} P_{n-i}$

Finally the image pixel value depends on whether it is lower than the t percent of average value of s pixel. If it is, we set it 1(black), otherwise 0 (white).

$\mathrm{T}(n)=\left\{\begin{array}{l}1 \text { if } P_{n}<\left(\frac{\mathrm{f}_{s}(n)}{s}\right)\left(\frac{100-t}{100}\right) \\ 0 \text { otherwise }\end{array}\right.$

Set s $1 / 8$ of image's width and $t 15$, and it seems that these value can produce good results for different image.

Considering the scanning mode, as shown in Fig. (6). as shown below:

In the previous solution we use the mean value, that is to say the weight of the scanned points to current point is the same. But according to the intuitive understanding, influence of the pixel closer to the current point is larger, and further is smaller. According to this problem we put forward $\mathrm{g}_{s}(n)$, and its significance are as follows:

$$
\begin{aligned}
\mathrm{g}_{\mathrm{s}}(n) & =g_{s}(n-1)-\frac{g_{s}(n-1)}{s}+P_{n} \\
& =P_{n}+\left(1-\frac{1}{s}\right) g_{s}(n-1) \\
& =P_{n}+\left(1-\frac{1}{s}\right) P_{n-1}+\left(1-\frac{1}{s}\right)^{2} P_{n-2} \ldots \ldots \\
& =\sum_{i=0}^{n}\left(1-\frac{1}{s}\right)^{i} P_{n-i}
\end{aligned}
$$

This description is more accurate, and it can be obtained by the addition and multiplication of recursive algorithm. On the other way its efficiency is relatively high.

Consider the information between lines, we set the threshold $h(n)$ value of current point the average value of current point $\mathrm{g}_{s}(n)$ and the above one $\mathrm{g}_{s}(n-$ width $)$

$\mathrm{h}(n)=\frac{g(n)+g(n-\text { width })}{2}$

The $\mathrm{g}_{n}$ was initialed as 127 , the intermediate values of 0 255 , here is the use of experience.

\subsubsection{Analysis of Experiment}

According to the principle and realization method of the above algorithm, the result of processing Fig. (1) and Fig. (3). are as follows:

Fig. (7) shows, the right black strip can be segmented clearly, but two extra lines are segmented too, duo to the most intensity of the right. When using the scanning method which from left to right and then from right to left, the gray value of image pixel closest to the light source on the left side of the pixel can make a big difference on the values of the current pixel. As shown in Fig. (8), it may produces some local strip; there are some empty holes in "Paperwork", and the gray line below is not segmented out; there are some empty holes in gray text part. These are all due to the using of experience value when set the $s$, $t$, which lead to unsatisfactory result when processing the subtle of the image.

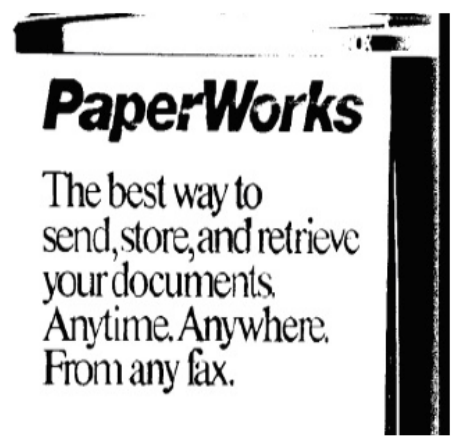

Fig. (7). Result1 of ODSA. 


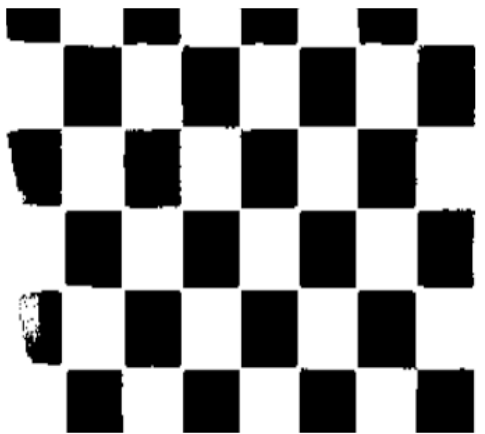

Fig. (8). Result2 of ODSA.

\subsection{The Improved Algorithm Based on Integration Im- age}

\subsubsection{The Principle and Implementation of the Improved Algorithm}

According to the Wellner algorithm's inherent problem which lead to unsmooth among the whole image and the problem that proposed in the above part which lead to not ideal in the subtle of the image, this paper presents a new adaptive segmentation algorithm. For the situation that the one-dimensional smoothing algorithm set the g0 127*s which lead to not ideal in local area of the image, this algorithm directly use the surrounding pixels color to produce the current pixel threshold value, it break the initial problem and the scanning sequence problem, and this kind of judgment is more scientific. First introduced the summed area tables (Summed-Area Table): Table $\mathbf{1}$ show the original table and Table $\mathbf{2}$ is the corresponding table of Table $\mathbf{1}$. To maintain a list which each element is the sum of the upper left corner of the positions of all elements:

In this algorithm, the value of pixels is from the surrounding pixel value. As shown in Fig. (9), assuming a $M^{*} N$ size image, the current pixel value is $\mathrm{f}(\mathrm{x}, \mathrm{y})$, using I $(\mathrm{x}, \mathrm{y})$ to represents the total value of the upper left pixel of the current pixel.

$\mathrm{S}_{m n}=\sum_{i=1}^{m} \sum_{j=1}^{n} t_{i j}$

Table 1. Original table.

\begin{tabular}{|l|l|l|l|}
\hline 2 & 3 & 2 & 1 \\
\hline 3 & 0 & 1 & 2 \\
\hline 1 & 3 & 1 & 0 \\
\hline 1 & 4 & 2 & 2 \\
\hline
\end{tabular}

Table 2. Integral table.

\begin{tabular}{|c|c|c|c|}
\hline 2 & 5 & 7 & 8 \\
\hline 5 & 8 & 11 & 14 \\
\hline 6 & 12 & 16 & 19 \\
\hline 7 & 17 & 23 & 28 \\
\hline
\end{tabular}

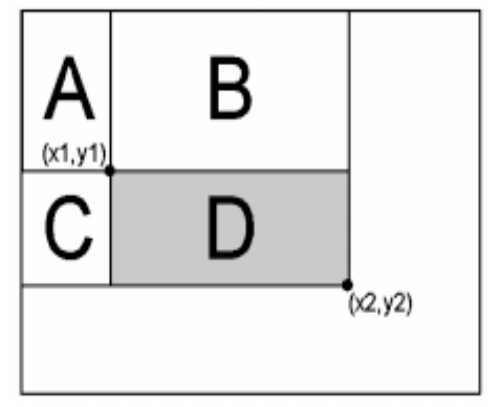

Fig. (9). Integral figure.

$\mathrm{I}(x, y)=f(x, y)+I(x-1, y)+I(x, y-1)-I(x-1, y-1)$

When the sum area table of the image are established, for any rectangular region, the upper left corner coordinates is $(\mathrm{x} 1, \mathrm{y} 1)$, the lower right corner coordinates is $(\mathrm{X} 2, \mathrm{Y} 2)$, then the area of the rectangular is:

$$
\begin{aligned}
& \sum_{x=x_{1}}^{x_{1}} \sum_{y=y_{1}}^{y_{2}} f(x, y)=I\left(x_{2}, y_{2}\right)-I\left(x_{2}, y_{1}-1\right)-I\left(x_{1}-1, y_{2}\right)+ \\
& I\left(x_{1}-1, y_{1}-1\right)
\end{aligned}
$$

According to Fig. (9), the area of region D can be expressed as:

$$
(A+B+C+D)-(A+B)-(A+C)+A
$$

\subsubsection{Analysis of Experiment}

According to the principle and realization method of the above algorithm, the result of processing Fig. (1) and Fig. (3) are as follows:

Comprehensive analysis: it can be seen from Fig. (10), that the text is no empty, the segmentation is clearly, the gray lines below "Paperworks" is clear, and the black bar on the right side is also revealed perfectly; From Fig. (10), we can see that when pass the segmented text to the OCR software to recognition, the recognition rate is up to $98 \%$. Fig. (11), shows the board under the uneven light of open office environment, and the segmentation effect is flawless.

According to the analysis of the visual effect of the above algorithm, this algorithm performance perfect in segmenting objects from the background. According to the the accuracy of OCR software recognition, the algorithm is a big help to subsequent treatment. According to the time complexity, after repeated testing, under the condition that the processor is AMD Athlon dual core, the frequency is $3.01 \mathrm{GHz}$, the memory is $2.00 \mathrm{~GB}$, and the image size is $2588 * 1940$, the result are as follows: the running time of Wellner algorithm is $504150 \mu \mathrm{s}$, the one of improved algorithm one is $97340 \mu \mathrm{s}$ and the one of the finally proposed Improved method is $56880 \mu \mathrm{s}$. According to the actual effect and the time complexity of the algorithm, the integral image algorithm is better than Wellner algorithm and the onedimensional smoothing algorithm.

\section{SUMMARY}

On the basis of the analysis of the classical Wellner algorithm we presents two improvements according to the inher- 


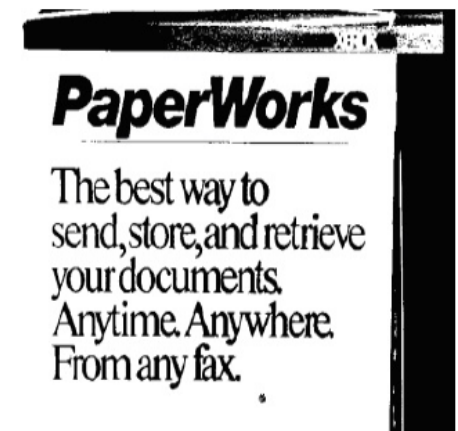

Fig. (10). Result 1 of integral image.

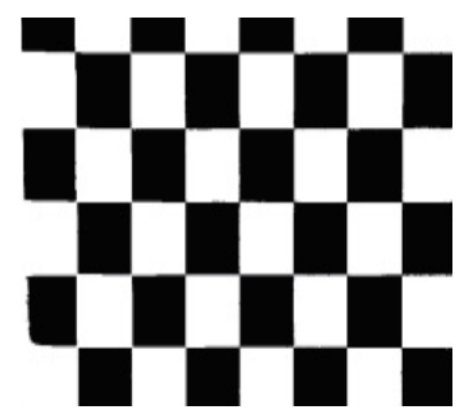

Fig. (11). Result 2 of integral image.

ent faults of the algorithm. One is one-dimensional smoothing algorithm. It desired to get more complete segmentation of the foreground prospect, to get more information of the foreground and proposed the considering of the spatial relations to smooth the image.Based on the first point we proposed the adaptive integral image algorithm. In order to get more detailed outlook, overcome the defects of the first two algorithms, we proposes dynamically set local threshold for different environment, to avoid the condition which is all black or all white of the local image, to segment object from background accurately. The foreground and background segmentation is clear, and the error rate is very low, and the time complexity is minimum, and it has good ability to adapt to the scene.Experiments show that, the integral image algorithm achieves good effect of image segmentation.

\section{CONFLICT OF INTEREST}

The authors confirm that this article content has no conflict of interest.

\section{ACKNOWLEDGEMENTS}

This work is supported by Natural Science Foundation of Hubei Province (No. 2014CFA528).

\section{REFERENCES}

[1] C. Guo, and Z. Hongfu, "2-D maximum entropy method of image segmentation based on genetic algorithm", Journal of ComputerAided Design and Computer Graphics, vol. 6, pp. 530-534, June 2002.

[2] J. He, H. Ge, and Y. Wang, "Survey on the methods of image segmentation research", Computer Engineering \& Science, vol. 12, pp. 58-60, July 2009.

[3] L. Jin, and L. Xia "Histgorm thresholding using index of fuzziness", Journal of Image and Graphics, vol. 5, pp. 390-395, May 2002.

[4] Y. Hao, and F. Zhu, "Fast algorithm for two-dmiensional otsu adaptive threshold algorithm", Journal of Image and Graphics, vol. 10, pp. 484-488, April 2005.

[5] F. H. Y. Chan, F. K. Lam, and H. Zhu, "Adaptive thresholding by variational method", IEEE Transactions on Image Processing, vol. 3, pp. 468-4737, March 1995.

[6] W. Zhi, and S. He, "An adaptive edge-detection method based on canny algorithm", Journal of Image and Graphics, vol. 9, pp 957962, August 2004.

[7] G. Liang, D. Liu, "Improvement of a two dimension adaptive thresholding segmentation algorithm", Computer Applications, vol. 5, pp. 43-47, May 2002.

[8] ImageShop.Wellner. Adaptive Binary Thresholding Algorithm[DB/OL]

http://www.cnblogs.com/Imageshop/archive/2013/04/22/3036127.h tml

Received: September 22, 2014

Revised: November 30, 2014

Accepted: December 02, 2014

(C) Daode et al.; Licensee Bentham Open.

This is an open access article licensed under the terms of the Creative Commons Attribution Non-Commercial License (http://creativecommons.org/licenses/by-nc/3.0/) which permits unrestricted, non-commercial use, distribution and reproduction in any medium, provided the work is properly cited. 